\title{
Discrete Geometry Applied in Hard Real-Time Systems Validation
}

\author{
Gaëlle Largeteau ${ }^{1}$, Dominique Geniet ${ }^{1}$, and Éric Andrès ${ }^{2}$ \\ 1 LISI, Université de Poitiers \& ENSMA, Téléport 2 - 1 avenue Clément Ader BP \\ 4010986961 Futuroscope Chasseneuil cédex, France. \\ 2 IRCOM-SIC, SP2MI, BP 30179, 86962 Futuroscope Cedex, France
}

\begin{abstract}
Off-line validation of hard real-time systems usually stands on state based models. Such approaches always deal with both space and time combinatorial explosions. This paper proposes a discrete geometrical approach to model applications and to compute operational feasability from topological properties. Thanks to this model, we can decide the feasability of real-time synchronous systems composed of periodic tasks, sharing resources, running on multiprocessor architectures. This method avoids state enumeration and therefore limits both space and time explosion: computing an automaton model takes at least 2 hours for a real application instead of at most 1 second using discrete geometry.
\end{abstract}

Keywords: Real-time, operational validation, multiprocessors, resource sharing, geometrical modeling.

\section{Introduction}

In a real-time system, the correctness of a computation depends on both the logical results of the computation and the time when results are produced. Time constraints are called strict if not respecting them involves irreparable consequences on the system safety. In this case, the system is called hard [But97. On the opposite, if not respecting deadlines keeps the system safe, the system is called soft. In this study, we only consider hard real-time systems where time constraints are strict.

A real-time system is a task set: each task is a process designed to react to an external incoming event. The systems we study use resources and run on centralized multiprocessor architectures. All processors are identical; tasks are preemptive and can move from a processor to another one at any time. Each task $\tau_{i}$ is specified by time characteritics: its first activation date $r_{i}$, its deadline $D_{i}$, its period $T_{i}$, and its execution time $C_{i}$ LL73. We assume that tasks are periodic and not reentrant: $\forall i \in[1, n], D_{i} \leq T_{i}$.

The operational validation of a real-time system is reached by proving that no task misses deadline, i.e by proving that there exists at least one time valid scheduling sequence for the system. This proof is obtained by feasability conditions or simulations. Validation is performed off-line for systems sharing re- 
sources which run on multiprocessor architectures, since there exists no necessary and sufficient feasability condition in this case Mok83. Off-line methods are usually based on state models (Petri nets, automata) ALU94 CHO96]. Each transition is associated with the same duration and constraints are expressed as numbers of transitions. Therefore time is discrete in these models. In [LG02, we have defined an implicit timed model, based on finite automata, that enumerates states of systems and therefore involves both space and time combinatorial explosion (about 2 h. 30 min. for 1000 states and 5000 transitions).

Observing the graphs of the automata we obtained in this approach, we have conceived a new model, based on discrete geometry, that is presented in this work. Our goal is to reduce notably both time and space combinatorial explosion in the validation process. In this model, we associate each task with a geometrical figure which only depends on time characteristics r, C, D, T. Geometrical operations (extrusion, cartesian product, intersection) allow to model concurrency and synchronization in a geometrical way. This model makes state enumeration implicit and therefore decreases both space and time combinatorial explosion while keeping a strong expression capacity (about $0.2 \mathrm{~s}$. for 1000 discrete points). We define the geometrical model for a single task in section 2 . In section 3, we present compositional operations to integrate both parallelism and synchronization in the model. Section 4 is dedicated to the presentation of a software implementing this modeling process.

\section{$2 \quad$ Model Definitions}

\subsection{A Two Dimensional Discrete Model to Represent Single Tasks}

A task is usually modeled by an automaton (see figure 10. Each transition of this automaton is associated with a duration of one time unit (time is discrete). A task state is defined by the execution progress $x=C(t)$ of the task and the total time $t$ since the system activation. Therefore, we consider, for each task, a two-dimensional space $(t, c): t$ adresses absolute time and $c$ adresses $x$.

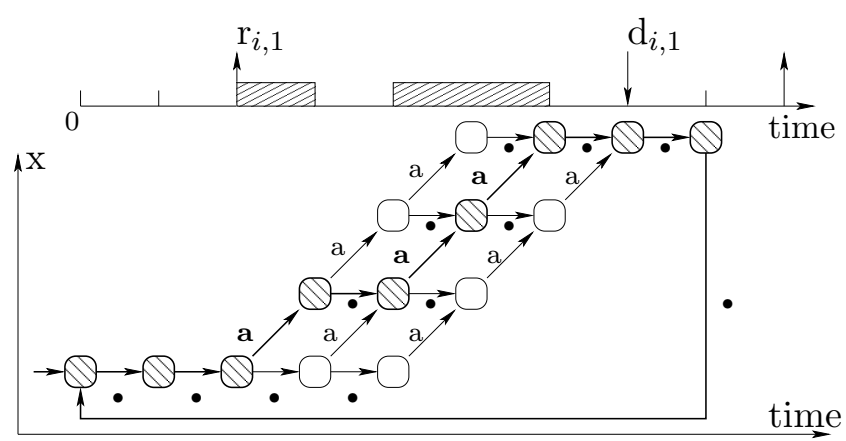

Fig. 1. Automaton model for task $\tau(\mathrm{r}=2, \mathrm{C}=3, \mathrm{D}=5, \mathrm{~T}=7)$ 


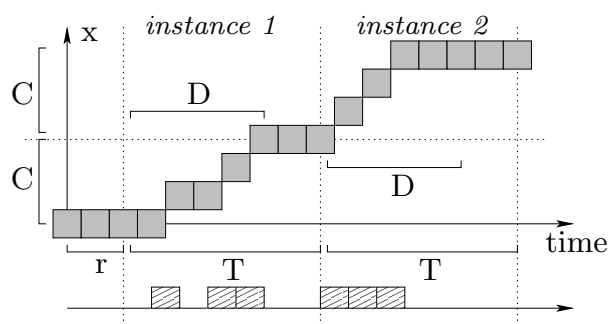

Fig. 2. A trace of task $\tau(\mathrm{r}=2, \mathrm{C}=3, \mathrm{D}=5, \mathrm{~T}=7)$

A task execution corresponds to the successive executions of its instances. At time $t, k$ instances of task $\tau(\mathrm{r}, \mathrm{C}, \mathrm{D}, \mathrm{T})$ are completed (corresponding to $k \times C$ time units of CPU owning) and the $(k+1)^{\text {th }}$ instance is running $\left(\mathrm{C}_{k+1}(\mathrm{t})\right.$ time units of past CPU owning). So, at time $t, C(t)=k \times C+C_{k+1}(t)$ time units have been executed. During its execution, an instance of task $\tau$ goes through several states whether it owns the CPU or not: the initial state $e_{0}$ of $\tau$ 's first instance is $(r, 0)$, its last state is $(r+T, C)$. The final state of any instance is the first state of the next instance. The final state $e_{k}$ of the $k^{\text {th }}$ instance of $\tau$ is $\left(e_{k-1}+(T, C)\right)$. We denote $r^{k}=r+k \times T$ the activation date of the $k^{\text {th }}$ task's instance of $\tau$.

A task execution is then totally defined by the set of all instance states. This set is the graph of a function in space $(t, c)$. This function is called "trace". Figure 2 is a discrete representation of the automaton sequence of figure 11. Note that for $\tau$, this function is not unique.

Let us now characterize traces. Since tasks cannot be parallelized, a task cannot be in more than one state at once and its state can not be undefined. Therefore, a task trace is a mapping between time and task execution progress. This mapping is an increasing function: either the task is progressing during execution; or the task is suspended and its execution progress state keeps the same value. Moreover, during any time interval $[\mathrm{t}, \mathrm{t}+1]$, no task can progress more than one step in one time unit since it is the maximal CPU time that can be allocated to the task for its execution during this interval.

Definition 1. We call trace of task $\tau$ an increasing mapping $\operatorname{Tr}(\tau)$ :

$$
\begin{aligned}
\operatorname{Tr}_{\tau}: \mathbb{Z}^{+} & \rightarrow \mathbb{Z}^{+} \\
t & \rightarrow \operatorname{Tr}_{\tau}(t) \text { such that } \forall t \geq 0, \operatorname{Tr}_{\tau}(t+1) \in\left\{\operatorname{Tr}_{\tau}(t), \operatorname{Tr}_{\tau}(t)+1\right\}
\end{aligned}
$$

Task $\tau$ must deal with its temporal characterisation (r,C,D,T): this property imposes geometrical constraints on execution traces of $\tau$ (see Figure 3). Some task traces are compatible with task operational charateristics: they are "valid task traces". Others are not compatible: they are unvalid. 


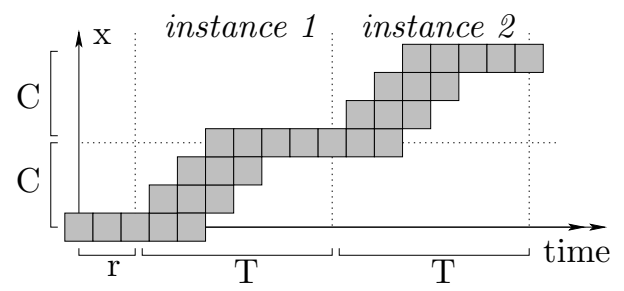

Fig. 3. Discrete model for two instances of task $\tau(\mathrm{r}=2, \mathrm{C}=3, \mathrm{D}=5, \mathrm{~T}=7)$

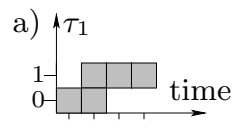

0123
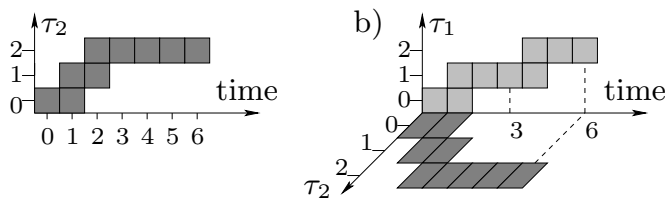

Fig. 4. Examples: $\tau_{1}(\mathrm{r}=0, \mathrm{C}=1, \mathrm{D}=2, \mathrm{~T}=3), \tau_{2}(\mathrm{r}=0, \mathrm{C}=2, \mathrm{D}=3, \mathrm{~T}=6)$

Definition 2. A valid trace of $\tau(r, C, D, T)$ is an execution trace $\operatorname{Tr}_{\tau} V$ such that:

$$
\begin{array}{ll}
\operatorname{Tr}_{\tau} V([0, r]) & =\{0\} \\
\operatorname{Tr}_{\tau} V\left(\left[r^{k}, r^{k}+D\right]\right) & =[(k-1) \times C, k \times C] \\
\operatorname{Tr}_{\tau} V\left(\left[r^{k}+D, r^{k+1}\right]\right) & =\{k \times C\}
\end{array}
$$

We call $T V(\tau)$ the set of $\tau$ 's valid traces. If $\tau$ misses no deadline, no instance of $\tau$ runs on time intervals $[0, \mathrm{r}]$ and $\left[\mathrm{r}^{k}+\mathrm{D}, \mathrm{r}^{k+1}\right]$. Therefore, traces $\operatorname{Tr}_{\tau} \mathrm{V}$ are constant functions over these intervals. The equation $\operatorname{Tr}_{\tau} \mathrm{V}\left(\mathrm{r}^{k}+\mathrm{D}\right)=\mathrm{k} \times \mathrm{C}$ means that the execution requirement $\mathrm{C}_{i}$ of the $\mathrm{k}^{\text {th }}$ instance of $\tau_{i}$ is completed before its deadline. Such a trace is a valid trace. We can then characterize the set $\Omega(\tau)$ which collects all points of valid traces.

Definition 3. The validity space $\Omega(\tau)$ of a task $\tau$ is:

$$
\Omega(\tau)=\bigcup_{\psi \in T V(\tau)}\{(t, \psi(t))\} .
$$

We note $T(\Omega(\tau))$ for $T V(\tau)$.

Figure 3 presents the validity space for the two first instances of a task. Each valid state of $\tau$ is associated with a point of $\mathbb{Z}^{2}$.

\subsection{Concurrency Modeling : $(n+1)$ Dimensional Discrete Model}

Let $\Gamma=\left(\tau_{i}\right)_{i \in[1, n]}$ be a set of tasks, designed to run concurrently. The state of $\Gamma$ at time $\mathrm{t}$ is defined by the states of all $\tau_{i}$. At time t, $\Gamma$ is valid if and only if all tasks of $\Gamma$ are valid. 
Definition 4. A valid state $P$ of a system $\Gamma$ at time $t$ is defined by:

$$
P=\left(t, x_{1}, \ldots, x_{n}\right) / \forall i \in[1, n],\left(t, x_{i}\right) \in \Omega\left(\tau_{i}\right)
$$

Therefore, the space of $\Gamma$ 's valid states is $(n+1)$-dimensional (see Figure 4).

$$
\Omega(\Gamma)=\left\{\left(t, x_{1}, \ldots, x_{n}\right) / \forall i \in[1, n]\left(t, x_{i}\right) \in \Omega\left(\tau_{i}\right)\right\}
$$

\subsection{Computing $\Omega(\Gamma)$}

We consider a system $\Gamma$ of $\mathrm{n}$ tasks.

\section{Geometrical Basic Notions And03]:}

Two discrete points $p$ and $q$ in $n$ dimensions are $k$-neighbour, for $0 \leq k \leq n$, if $\forall 1 \leq i \leq n,\left|p_{i}-q_{i}\right| \leq 1$ and if $k \leq n-\sum_{i=1}\left|p_{i}-q_{i}\right|$. A $k$-path in a discrete object $A$ is a discrete $A$ point list such that two consecutive points of this list are $k$-neighbour (a task trace is a 0 -path in 2 -dimension). If there exists a $k$ path in a discrete object $A, A$ is said $k$-connected. A $k$-component is a maximal $k$-connected discrete object.

Definition 5. Let $\mathcal{I}=\left\{i_{1}, \ldots, i_{|\mathcal{I}|}\right\} \quad\left(i_{1}<i_{2}<\ldots<i_{|\mathcal{I}|}\right)$. We define the injection operation $\mathcal{J}_{\mathcal{I}, n}$ in the following way: $\mathcal{J}_{\mathcal{I}, n}: \mathbb{Z}^{|\mathcal{I}|} \rightarrow \mathbb{Z}^{n+1}$

$$
\left(x_{1}, \ldots, x_{|\mathcal{I}|}\right) \rightarrow \overbrace{\left(0, \ldots, 0, x_{1}, 0, \ldots, 0, x_{j}, 0, \ldots, 0, x_{|\mathcal{I}|}, 0, \ldots, 0\right)}^{n+1}
$$

Notations:

- $\mathcal{J}_{i, n}=\mathcal{J}_{\{1, i+1\}, n}:(\mathrm{a}, \mathrm{b}) \rightarrow \underset{1}{(\mathrm{a}, 0, . ., 0, \mathrm{~b}, 0 \ldots, 0)}$

- $\Lambda_{\mathcal{I}, n}$ is the following cartesian product:

$$
\Lambda_{\mathcal{I}, n}=\mathbb{Z}^{i_{1}-1} \times\{0\} \times \mathbb{Z}^{i_{1}-i_{2}-1} \times\{0\} \times \ldots \times \mathbb{Z}^{i_{|\mathcal{I}|}-i_{|\mathcal{I}|-1}-1} \times\{0\} \times \mathbb{Z}^{n+1-i_{|\mathcal{I}|}} .
$$

- $\Lambda_{i, n}$ is the cartesian product $\Lambda_{i, n}=\{0\} \times \mathbb{Z}^{i-1} \times\{0\} \times \mathbb{Z}^{n-i}=\Lambda_{\{1, i+1\}, n}$.

Definition 6. We define the interleaved cartesian product $\mathcal{J}_{\mathcal{I}, n}^{\Lambda}: \mathbb{Z}^{|\mathcal{I}|} \rightarrow \mathcal{P}\left(\mathbb{Z}^{n+1}\right)$ $\left(x_{1}, \ldots, x_{|\mathcal{I}|}\right) \rightarrow\left\{\mathcal{J}_{\mathcal{I}, n}\left(\left(x_{1}, \ldots, x_{|\mathcal{I}|}\right)\right)+\lambda, \lambda \in \Lambda_{\mathcal{I}, n}\right\}$

Notations:

- $\mathcal{J}_{i, n}^{\Lambda}=\mathcal{J}_{\{1, i+1\}, n}^{\Lambda}=\left\{\mathcal{J}_{i, n}((a, b))+\lambda, \quad \lambda \in \Lambda_{i, n}\right\}$.

Definition 7. We call "concurrent product" (denoted $\otimes$ ) of $\Omega\left(\tau_{1}\right)$ and $\Omega\left(\tau_{2}\right)$ the set $\Omega\left(\tau_{1}\right) \otimes \Omega\left(\tau_{2}\right)=\mathcal{J}_{1, n}^{\Lambda}\left(\Omega\left(\tau_{1}\right)\right) \cap \mathcal{J}_{2, n}^{\Lambda}\left(\Omega\left(\tau_{2}\right)\right)$. 


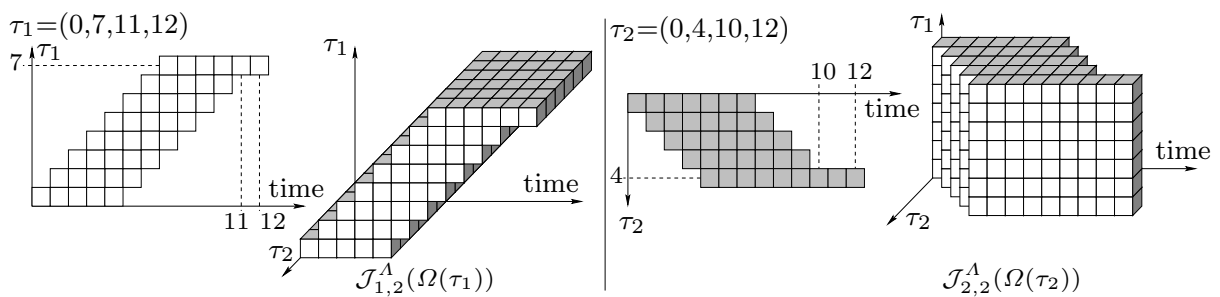

Fig. 5. Example: $\mathcal{J}_{1,2}^{\Lambda}\left(\Omega\left(\tau_{1}\right)\right)$ and $\mathcal{J}_{2,2}^{\Lambda}\left(\Omega\left(\tau_{2}\right)\right)$

\section{Remarks:}

-The operation $\otimes$ is associative, therefore we can generalize the notation:

$$
\Omega\left(\tau_{1}, \ldots, \tau_{n}\right)=\bigotimes_{i=1}^{i=n} \Omega\left(\tau_{i}\right)
$$

- $\forall \mathcal{J} \in \mathcal{J}_{i, n}^{\Lambda}((a, b)), \forall k \in[1, i-1] \cup[i+1, n], \exists x_{k} \in \mathbb{Z}$ such that:

$$
\mathcal{J}=\left(\mathbf{a}, x_{1}, \ldots, x_{i-1}, \mathbf{b}, x_{i+1}, \ldots, x_{n}\right)
$$

- Projection $\Pi_{i}:\left(\mathrm{y}_{1}, \ldots, \mathrm{y}_{i+1}, \ldots, \mathrm{y}_{n+1}\right) \rightarrow\left(\mathrm{y}_{1}, \mathrm{y}_{i+1}\right)$ is a reverse operation of $\mathcal{J}_{i, n}^{\Lambda}$ : $\Pi_{i}\left(\mathcal{J}_{i, n}^{\Lambda}(\mathrm{a}, \mathrm{b})\right)=\{(\mathrm{a}, \mathrm{b})\}$.

The following theorem says that the $(n+1)$ dimensional discrete model is obtained as an intersection of our interleaved cartesian product. It provides a direct algorithm.

Theorem 1. $\Omega(\Gamma)=\bigotimes_{i=1}^{i=n} \Omega\left(\tau_{i}\right)$.

Proof: Let us show that $\bigotimes_{i=1}^{i=n} \Omega\left(\tau_{i}\right) \subset \Omega(\Gamma)$ :

Let $\mathrm{P}=\left(t, x_{1}, x_{2}, \ldots x_{n}\right) \in \bigotimes_{i=1}^{i=n} \Omega\left(\tau_{i}\right)$. The definition of $\otimes$ gives:

$\mathrm{P}=\left(t, x_{1}, x_{2}, \ldots x_{n}\right) \in \bigcap_{i \in[1, n]}\left(\mathcal{J}_{i, n}^{\Lambda}\left(\Omega\left(\tau_{i}\right)\right)\right)$. Since $\mathrm{P}$ belongs to an intersection set, we get: $\forall i \in[1, n], P \in \mathcal{J}_{i, n}^{\Lambda}\left(\Omega\left(\tau_{i}\right)\right)$. Using the two-dimensional projection on $\tau_{i}$-space $\left(\mathrm{t}, \tau_{i}\right): \forall i \in[1, n], \Pi_{i}(P) \in \Pi_{i}\left(\mathcal{J}_{i, n}^{\Lambda}\left(\Omega\left(\tau_{i}\right)\right)\right)=\Omega\left(\tau_{i}\right)$. And then $\forall i \in[1, n],\left(t, x_{i}\right) \in \Omega\left(\tau_{i}\right)$. Therefore, $P \in \Omega(\Gamma)$.

Let us show that $\Omega(\Gamma) \subset \bigotimes_{i=1}^{i=n} \Omega\left(\tau_{i}\right)$ :

Let $\mathrm{P}=\left(t, x_{1}, x_{2}, \ldots x_{n}\right) \in \Omega(\Gamma)$. We consider the projection of $\mathrm{P}$ on each plane $\left(\mathrm{t}, \tau_{i}\right): \forall i \in[1, n] \Pi_{i}(P) \in \Omega\left(\tau_{i}\right)$. We then consider $\mathcal{J}_{i, n}^{\Lambda}$ for each $\Pi_{i}(\mathrm{P})$ : $\forall i \in[1, n] \mathcal{J}_{i, n}^{\Lambda}\left(\Pi_{i}(P)\right) \subset \mathcal{J}_{i, n}^{\Lambda}\left(\Omega\left(\tau_{i}\right)\right)$. Finally, we consider the intersection of all sets we have obtained: 


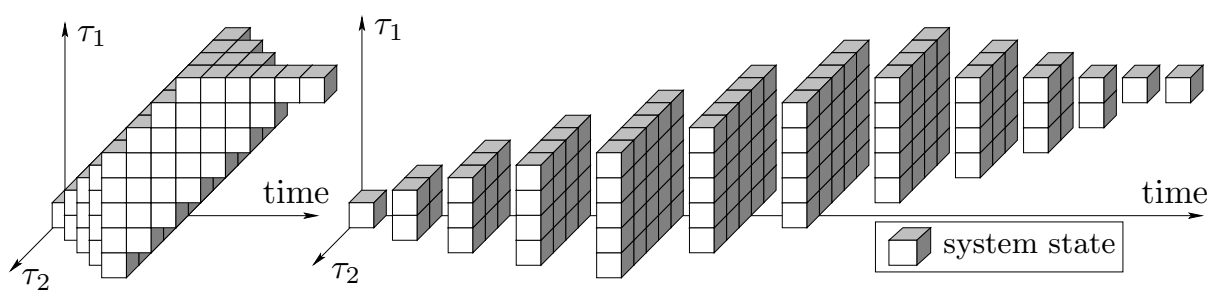

Fig. 6. Example: geometrical modeling for a two tasks system, $\Omega\left(\tau_{1}\right) \otimes \Omega\left(\tau_{2}\right)$

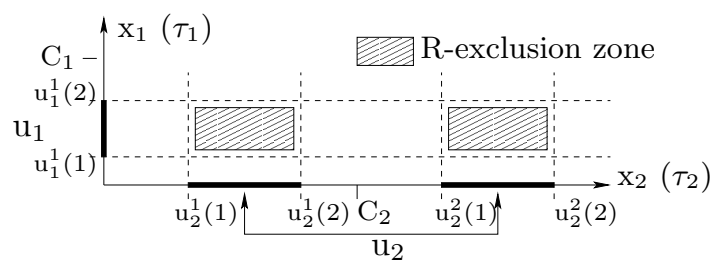

Fig. 7. Example: both $\tau_{1}$ and $\tau_{2}$ use resource $\mathrm{R}$

$$
\begin{aligned}
& \bigcap_{i \in[1, n]}\left(\mathcal{J}_{i, n}^{\Lambda}\left(\Pi_{i}(P)\right)\right) \subset \bigcap_{i \in[1, n]}\left(\mathcal{J}_{i, n}^{\Lambda}\left(\Omega\left(\tau_{i}\right)\right)\right) \text { Since } \bigcap_{i \in[1, n]}\left(\mathcal{J}_{i, n}^{\Lambda}\left(\Pi_{i}(P)\right)\right)= \\
& \left\{\left(t, x_{1}, x_{2}, \ldots x_{n}\right)\right\}=\{P\}, \text { we get: } P \in \bigotimes_{i=1}^{i=n} \Omega\left(\tau_{i}\right) .
\end{aligned}
$$

\subsection{Synchronization Integration}

In real-time systems, tasks use critical resources and the processor number is limited. In next parts, we integrate these constraints in our modelisation.

\section{Resource Sharing}

Resources must be used in mutual exclusion : only one task can use resource $\mathrm{R}$ at time t. Therefore, some states of $\Omega(\Gamma)$ become invalid from the resource sharing point of view. On a geometric point of view, the $\mathrm{k}^{\text {th }}$ instance of a task corresponds to the task execution progress interval $\left[\mathrm{C}_{i} \times \mathrm{k}, \mathrm{C}_{i} \times \mathrm{k}+\mathrm{C}_{i}\right]$. We define $\mathrm{u}^{k}(1)$ and $\mathrm{u}^{k}(2)$ such that this instance uses $\mathrm{R}$ during the execution time interval: ] $\mathrm{u}_{i}^{k}(1), \mathrm{u}_{i}^{k}(2)\left[\subset\left[\mathrm{C}_{i} \times \mathrm{k}, \mathrm{C}_{i} \times \mathrm{k}+\mathrm{C}_{i}\right]\right.$.

We denote by $\mathrm{u}_{i}$ the time interval union $\left.\bigcup_{k \in \mathbb{N}}\right] \mathrm{u}_{i}^{k}(1), \mathrm{u}_{i}^{k}(2)[$, corresponding to all resource requirement intervals for all instances of $\tau_{i}$ (see Figure 7).

Since a task cannot own the resource if another task already uses it, two tasks cannot be in $\mathrm{R}$ critical section at the same time. Then if $\mathrm{P}=\left(\mathrm{t}, x_{1}, \ldots, x_{n}\right)$ and $x_{i}$ $\in u_{i}$, we must get $\forall j \neq i, x_{j} \notin u_{j}$. Therefore, for a valid state $\mathrm{P}=\left(t, x_{1}, \ldots, x_{n}\right)$ of $\Gamma$, the following property stands: $\left|\left\{x_{i} / x_{i} \in u_{i}\right\}\right| \leq 1$. The validity space of $\Gamma$ respecting resource $\mathrm{R}$ sharing is (see figure 8 ): 


$$
\Omega_{R}(\Gamma)=\left\{\left(t, x_{1}, \ldots, x_{n}\right) \in \Omega(\Gamma) /\left|\left\{x_{i} / x_{i} \in u_{i}\right\}\right| \leq 1\right\}
$$

Here, we deal only with one resource R. For many resources, since resources are independant, the technique can be applied by induction. Let $\Gamma_{R}=\left(\tau_{j}\right)_{j \in \mathcal{I}_{R}}$ be the set of tasks sharing resource $\mathrm{R}$ (we note $\mathcal{I}_{R}$ the set of indices of tasks sharing $\mathrm{R})$. The states of $\Gamma_{R}$ which are associated with a simultaneous use of $\mathrm{R}$ are unvalid. The R-exclusion zone $\Omega(R)$ of $\mathrm{R}$ collects all states of $\Gamma_{R}$ corresponding $\mathrm{R}$ misuses. $\Omega(R)$ is part of the subspace associated with all tasks sharing $\mathrm{R}$, since it implies the simultaneous run of at least two of these tasks.

Definition 8. The R-exclusion zone is (see Figure 7):

$$
\Omega(R)=\left\{\left(x_{i}\right)_{i \in \mathcal{I}_{R}} /\left|\left\{x_{i} / x_{i} \in u_{i}\right\}\right|>1\right\} .
$$

A state of $\Omega(R)$ only concerns tasks of $\Gamma_{R}$. A state $\mathrm{s}=\left(\mathrm{t}, x_{1}, \ldots, x_{n}\right)$ is unvalid if $\Pi_{\Gamma_{R}}(s) \in \Omega(R)$. Therefore, the set $\eta_{R}=\left\{\left(t, x_{1}, \ldots, x_{n}\right) / t \in \mathbb{Z},\left|\left\{x_{i} / x_{i} \in u_{i}\right\}\right|>\right.$ $1\}$ of unvalid states can be obtained thanks to a concurrent cartesian product and an extrusion operation.

Theorem 2. $\eta_{R}=\operatorname{Extr}\left(\mathbb{Z}, \mathcal{J}_{\mathcal{I}_{\mathcal{R}, n}}^{\Lambda}(\Omega(R))\right)$.

Proof: This theorem comes directly from the definitions of $\Omega(\mathrm{R})$, the extrusion operation and interleaved cartesian product operation. $\Omega(\mathrm{R})$ collects all unvalid states of $\Gamma_{R}$ : it is a $\left|\mathcal{I}_{R}\right|$-dimensional object. The interleaved cartesian product associates these states with all possible states of $\Gamma \backslash \Gamma_{R}$. Then all states of $\Gamma$ that are unvalid in the R-sharing point of view are reached. $\mathcal{J}_{\mathcal{I}_{\mathcal{R}}, n}^{\Lambda}(\Omega(R))$ is an n-dimensional object. Now one must integrate that these states are always unvalid. This is done by extruding this objet following the time direction $(\mathbb{Z})$. This operation collects all R-sharing unvalid states of $\Gamma$. Then we get $\eta_{R}=\operatorname{Extr}\left(\mathbb{Z}, \mathcal{J}_{\mathcal{I}_{\mathcal{R}, n}}^{\Lambda}(\Omega(R))\right)$.

All states of $\eta_{R}$ are not valid from the resource sharing point of view. Valid states of the application are then in $\Omega(\Gamma)$ but not in $\eta_{R}$.

Theorem 3. $\Omega_{R}(\Gamma)=\Omega(\Gamma) \backslash \eta_{R}$.

Therefore, the set of valid traces including resource sharing is:

$T\left(\Omega_{R}(\Gamma)\right)=\left\{\psi \in T(\Omega(\Gamma)) / \forall t \in \mathbb{Z}^{+}, \psi(t)=\left(x_{1}, \ldots, x_{n}\right),\left(t, x_{1}, \ldots, x_{n}\right) \in \Omega_{R}(\Gamma)\right\}$

\section{Processor Sharing}

While building $\Omega_{R}(\Gamma)$, we have not considered the number of processors. However, this parameter makes each trace $\omega$ in $\mathrm{T}_{R}(\Gamma)$ valid or unvalid according to the minimal number of processors useful to execute $\omega$.

During an execution, the number of active processors is constant between two consecutive context switches. To decide the validity of a trace, we only have to look at it at context switch times. Let us note by q the scheduling quantum. If there are $k$ running tasks between two given context switches $a$ and $b$, the trace is called $k$-concurrent between $a$ and $b$. 


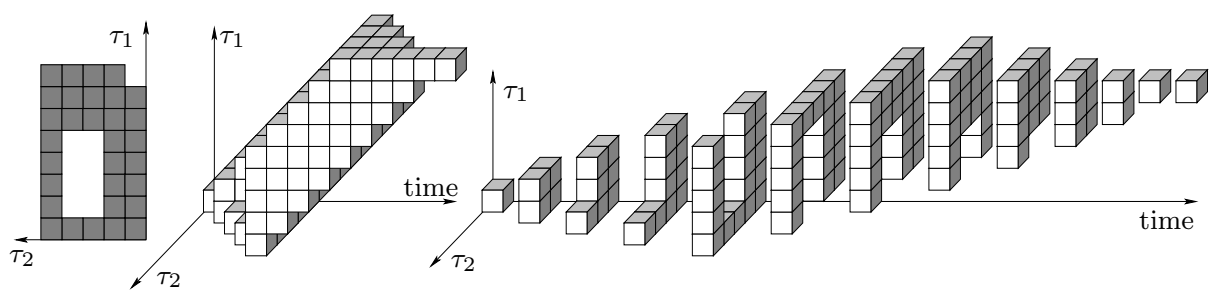

Fig. 8. Geometrical model of a system including resource sharing

Definition 9. A trace $\psi \in T\left(\Omega_{R}(\Gamma)\right)$ is $k$-concurrent between two context switch times $i \times q$ and $(i+1) \times q$ if and only if: $\psi(i \times q)=\left(i \times q,\left(x_{i}\right)_{i \in[1, n]}\right)$ and $\psi((i+1) \times q)$ $=\left((i+1) \times q,\left(y_{i}\right)_{i \in[1, n]}\right) \Rightarrow \sum_{i \in[1, n]}\left|x_{i}-y_{i}\right| \leq k \times q$.

We said that discrete points $\psi(i \times q)$ and $\psi((i+1) \times q)$ are $k$-concurrent. This definition of $k$-concurrency in a $n+1$ dimensional space corresponds to the definition of the $n-k$-neighbourhood. A $k$-concurrent trace is then a $n-k$-path in $\Omega_{R}(\Gamma)$. We denote by $\mathrm{T}_{R, k}(\Gamma)$ the set of $k$-concurrent traces of $\mathrm{T}\left(\Omega_{R}(\Gamma)\right)$.

Definition 10. A set $\Omega_{R}(\Gamma)$ is $k$-concurrent if there exists at least one $k$ concurrent trace $\psi$ in $T_{R}(\Gamma)$.

Remark: If a set $\Omega_{R}(\Gamma)$ is $k$-concurrent, then it is a $n-k$-component and there exists at least one valid sheduling sequence for $\Gamma$ on a $k$ processor architecture.

\subsection{Feasability Decision}

For a system running on a $k$ processor architecture and sharing a resource $R$, a valid scheduling is a $k$-concurrent trace in $\mathrm{T} \Omega_{R}(\Gamma)$. The feasability decision is reached by evaluating the predicate: $T_{R, k}(\Gamma) \neq \emptyset$.

\section{Implementation}

We have developed the software GemSMARTS (Geometric Scheduling Modeling and Analysis of Real-Time Systems) which computes the set $\Omega_{R}(\Gamma)$. We have tested a discrete data structure implemented through classical matrices. Since we avoid enumeration, we get more efficient computing times : using automata, models are obtained after at least 2 hours instead of 1 second at most using discrete geometry.

Figure 9 shows $\Omega_{R}(\Gamma)$ for a two-tasks system sharing a resource.

While avoiding enumeration in the discrete model, we reach very efficient computation time. As a comparison, for a seven task system sharing four resources, computing the automaton model takes more than 2 hours while the computation of the discrete modele last less than 1 second. 


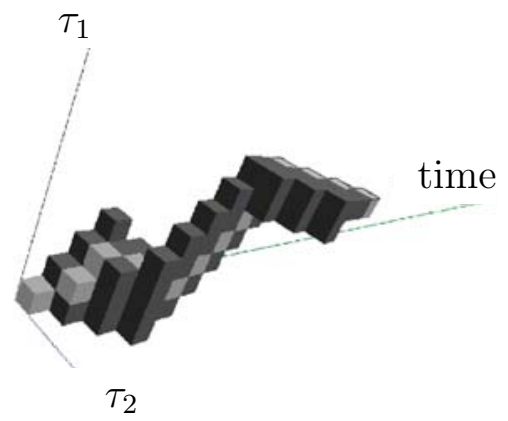

Fig. 9. System geometrical model: $\tau_{1}=(0,7,10,12), \tau_{2}=(0,3,6,6)$

\section{Conclusion}

Validity spaces are useful to model hard real-time systems running on multiprocessor architectures and sharing resources. Feasability of task systems and optimal numbers of processors can be computed thanks to the k-concurrency concept.

Feasability is usually decided using state based model and model checkers LG02]. Using validity spaces involves a noteworthy improvement: the time saved on a time free automata model is about $85 \%$. The data structure we have tried (matrices) is useful to implement our model and developed algorithms are already more efficient than the previous ones (using automata) although they are far from being optimized. We have reached a limited time complexity depending mainly on the number of tasks whereas classical models follow complexities which depend on time. This method allows to drive back both space and time explosion.We are studying both space and time complexities for optimized version of the geometrical algorithms.

Ongoing works concern definitions of both topological and geometrical properties to precisely characterize scheduling sequences. We plan, for example, to define topological properties for validate on-line classical scheduling (RM, ED, and so on), in order to propose multiprocessor versions of on-line validation techniques integrating resource sharing.

\section{References}

[ALU94] R.Alur, D.Dill: A theory of timed automata. Theoretical Computer Science, vol. 126, pp 183-235.(1994).

[And03] E.Andres: Discrete linear objects in dimension n: the standard model. Graphical Models 65(1-3), pp 92-111. (2003).0

[But97] G. Buttazzo: Hard real-time computing systems: Predictable scheduling algorithms and applications. Kluwer Academic Publishers. (1997).

[CHO96] A.Choquet-geniet, D.Geniet, F.Cottet: Exhaustive computation of scheduled task execution sequences of real-time application. Proc FTRTFT'96.(1996) 
[LL73] C.L. Liu et J.W. Layland : Scheduling algorithms for multiprogramming in real-time environnement. Journal of the ACM, vol 20, pp 46-61.(1973)

[LG02] G.Largeteau, D.Geniet: Term Validation of Distributed Hard Real-time Applications. Conference on Implementation and Application of Automata. (2002).

[Mok83] A.K.Mok: Fundamental design problems for the hard real-time environments. Ph.D. MIT.(1983). 